Hung Nhon Vuong, Department of Mathematics, University of California, Santa Barbara, CA 93106., e-mail: cooper@math.ucsb.edu

\title{
MEASURE LINEARITY OF BILIPSCHITZ MAPS OF SELF-SIMILAR CANTOR SETS
}

\begin{abstract}
Let $C$ and $C^{\prime}$ be Cantor sets in $\mathbb{R}^{n}$ generated by Euclidean similarities, called clone Cantor sets. There are associated Hausdorff measures $\mu_{C}$ and $\mu_{C^{\prime}}$. We show that if there is a bi-Lipschitz map $\phi$ of $\mathbb{R}^{n}$ which maps $C$ onto a clopen subset of $C^{\prime}$ then there exists a constant $\lambda>0$ and a subset $A$ of $C$ with $\mu_{C}(A)>0$ and such that for all $\mu_{C}$-measurable sets $B$ of $A$ we have $\phi(B)$ is $\mu_{C^{\prime}}$-measurable and $\mu_{C^{\prime}}(\phi B)=\lambda \mu_{C}(B)$. This result leads to an almost complete classification of clone Cantor sets up to bi-Lipschitz maps of Euclidean space.
\end{abstract}

A subset $C$ of $\mathbb{R}^{n}$ is a Cantor set if it is compact, totally disconnected, and perfect. When $n$ is greater than 2 the geometry of Cantor sets in $\mathbb{R}^{n}$ can be extremely complex. A Cantor set in $\mathbb{R}^{n}$ is tame if there exists a homeomorphism $h: \mathbb{R}^{n} \rightarrow \mathbb{R}^{n}$ such that $h(C)$ lies on a Euclidean straight line; otherwise $C$ is wild. Two Cantor sets in $\mathbb{R}^{n}$ are topologically equivalent if there is a homeomorphism $h: \mathbb{R}^{n} \rightarrow \mathbb{R}^{n}$ such that $h(C)=C^{\prime}$. It is well known that any two tame Cantor sets in $\mathbb{R}^{n}$ are topologically equivalent; moreover, for $n=1$ or 2 all Cantor sets in $\mathbb{R}^{n}$ are tame. An example of a wild Cantor set in $S^{3}$ was first constructed by L. Antoine in 1921. In this paper, we examine a subclass of the tame Cantor sets, the class of clone Cantor sets (defined below) from the perspective of bi-Lipschitz maps. A clone Cantor set is self-similar by similarity maps of $\mathbb{R}^{n}$. The tool we will be using is the Hausdorff measure associated with these clone Cantor sets.

One finds a variety of definitions of the notion of self-similarity in mathematical literature. While these definitions may differ slightly, they all contain

\footnotetext{
Mathematical Reviews subject classification:

Received by the editors June 9, 1994

* The results presented here are from the author's Ph.D. dissertation written under the direction of Daryl Cooper.
} 
a similar idea: Geometrically, a set is self-similar if it is a union of smaller copies of itself. For our work, we use the following definition which is found in [5]. A nonempty compact subset $K$ of a separable complete metric space $X$ is said to be self-similar with respect to $\left\{f_{1}, f_{2}, \ldots, f_{m}\right\}$, where each $f_{i}$ is a contraction on $X$, if $K$ satisfies the set equation

$$
K=f_{1}(K) \cup f_{2}(K) \cup \cdots \cup f_{m}(K) .
$$

We study self-similar Cantor sets which are self-similar with respect to a specific type of contraction: a similarity of $\mathbb{R}^{n}$. A clone Cantor set is a maximal invariant set for the semigroup of similarities generating the set.

The results of this paper are a generalization of results of [2], which classified the clone Cantor sets on the line up to bi-Lipschitz equivalence.

This paper is divided into two sections. Section 1 contains various definitions as well as a construction of these clone Cantor sets. A brief analysis of the Hausdorff measure and dimension of these clone Cantor sets is also given in this section. The reader may refer to [8] or [4] for a full discussion of Hausdorff measure and dimension. Section 2 contains our main result. This says that a bi-Lipschitz map from one clone Cantor set to another, when restricted to a suitable clopen, is linear at the level of measure theory. More precisely, if $C$ and $C^{\prime}$ are clone Cantor sets in $\mathbb{R}^{n}$ and $\phi: \mathbb{R}^{n} \rightarrow \mathbb{R}^{n}$ is a bi-Lipschitz map such that $\phi(C)$ is a clopen subset of $C^{\prime}$, then $\phi$ induces a bi-Lipschitz map $\Phi$ of $\mathbb{R}^{n}$ which maps $C$ onto a clopen subset of $C^{\prime}$. Furthermore $\Phi$ has constant Radon-Nikodym derivative with respect to the associated Hausdorff measures on $C$ and $C^{\prime}$. This result leads to the development of an invariant for these clone Cantor sets under bi-Lipschitz equivalence called the clopen invariant. This is the equivalence class of the countable set of measures of the clopens of a clone Cantor set. The equivalence relation is re-scaling. Ultimately, our goal is to classify all clone Cantor sets of $\mathbb{R}^{n}$ up to bi-Lipschitz equivalence. We accomplish this task for "most" of these Cantor sets in [9]. A summary of which is given in Theorem 1.4.

The author thanks the referee for helpful comments, and for suggesting a broader definition of clone Cantor set than the original. Many of the results in this paper have been extended to metric Cantor sets, not necessarily subsets of Euclidean space, but possessing a certain self-similarity structure. This was used in the study of the quasi-geometry of certain Baumslag-Solitar groups [1]. 


\section{Preliminaries}

The class of Cantor sets we are about to examine has a self-similarity structure arising from the dynamical systems which produce these sets. We may view this class as a generalization of the middle third Cantor set on the line to Cantor sets in $\mathbb{R}^{n}$. We introduce a method for generating these clone Cantor sets.

\subsection{Construction of clone Cantor Sets}

The following dynamical system, called a clone structure decomposition, has as its maximal invariant set a clone Cantor set:

Let $s: \mathbb{R}^{n} \rightarrow \mathbb{R}^{n}$ be a similarity, i.e., there exists a constant $c>0$ such that $|s(x)-s(y)|=c|x-y|$ for all $x, y$ in $\mathbb{R}^{n}$. Then $s$ can be expressed as

$$
s(x)=\lambda T(x)+\vec{x}_{0} \quad \text { for some } \lambda \in \mathbb{R}^{+}, T \in O(n), \text { and } \vec{x}_{0} \in \mathbb{R}^{n} .
$$

A proof of (1) can be found in [6]. Next, let $\mathcal{I}$ be a compact, connected subset in $\mathbb{R}^{n}$. For each $i$ with $1 \leq i \leq q$, suppose that $\tau_{i}$ is an expanding similarity of $\mathbb{R}^{n}$ and suppose that $A_{i} \equiv \tau_{i}^{-1} \mathcal{I}$ is a proper subset of $\mathcal{I}$. Informally we say that $A_{i}$ is a smaller copy of $\mathcal{I}$ contained inside $\mathcal{I}$. Suppose that $A_{1}, A_{2}, \ldots, A_{q}$ are pairwise disjoint. Define

$$
\tau \equiv \bigcup_{i=1}^{q} \tau_{i}: \bigcup_{i=1}^{q} A_{i} \rightarrow \mathcal{I}
$$

The Cantor set generated by the dynamical system $\tau$, is denoted $C(\tau)$, (or simply $C$ when no confusion arises) is

$$
C(\tau)=\bigcap_{k=0}^{\infty} \tau^{-k}(\mathcal{I})
$$

where $\tau^{0}$ is the identity map and $\tau^{-k}$ is the $k$-fold composition

$$
\overbrace{\tau^{-1} \circ \tau^{-1} \circ \cdots \circ \tau^{-1}}^{k \text { times }} \text {. }
$$

Remark 1.1. The following are obvious:

- $C$ is the maximal invariant set of the map $\tau$.

- According to the aforementioned definition of self-similarity, $C$ is selfsimilar with respect to $\left\{\tau_{1}^{-1}, \tau_{2}^{-1}, \ldots, \tau_{q}^{-1}\right\}$. 
Definition 1.2. The maps $\tau_{1}, \tau_{2}, \ldots, \tau_{q}$ are called level-1 clone maps of $C$. The components of $\tau^{-k}(\mathcal{I})$ are called level- $k$ clones of $C$; and if $A$ is a level- $k$ clone of $C$ and $B \subseteq A$ is a level- $(k+l)$ clone of $C$, we call $B$ a (relative) level-l clone of $A$. Some authors use the term cylinder in place of clone. We consider $\mathcal{I}$ to be the level-0 clone of $C$.

Thus the sets $A_{1}, A_{2}, \ldots, A_{q}$ are the level- 1 clones of $C$. From the above definition, it follows that each level- $k$ clone of $C$ is a small copy of $\mathcal{I}$. On the other hand, if $A$ is a level- $k$ clone of $C$, then the map $\left.\tau^{k}\right|_{A}$ maps $A$ onto $\mathcal{I}$ so that the subset of $C$ contained in $A$ is mapped onto $C$. From this perspective, the subset of $C$ contained in $A$ is just a linearly scaled down copy of $C$. This illustrates the self-similarity structure of $C$. Because of this property, we sometimes think of a clone $A$ of $C$ as the subset of $C$ contained in $A$. This abuse of notation will always be clear in its context. Furthermore, note that the map $\left.\tau^{k}\right|_{A}$ may be written uniquely as a $k$-fold composition $\tau_{i_{1}} \circ \tau_{i_{2}} \circ \cdots \circ \tau_{i_{k}}$, where $1 \leq i_{j} \leq q$, of level- 1 clone maps. In this light, we say that $C$ is generated by the maps $\tau_{1}, \tau_{2}, \ldots, \tau_{q}$ and refer to the $k$-fold composition $\left.\tau^{k}\right|_{A}$ as a level- $k$ clone map.

Notation: The collection of maps $\left\{\tau_{1}, \tau_{2}, \ldots, \tau_{q}\right\}$ together with $\mathcal{I}$ uniquely determine a clone Cantor set $C$. We will adopt the notation $C(\tau)=\left\{\tau_{i}\right\}_{i=1}^{q}$ to indicate the clone Cantor set $C(\tau)$ generated by the collection of level-1 clone maps $\left\{\tau_{i}\right\}_{i=1}^{q}$ and suppress the set $\mathcal{I}$ in this notation.

A map $\phi: \mathbb{R}^{n} \rightarrow \mathbb{R}^{n}$ is a bi-Lipschitz map if there exist a bi-Lipschitz constant $K \geq 1$ such that for all $x \neq y$ in $\mathbb{R}^{n}$, the following inequality is satisfied:

$$
K^{-1} \leq \frac{|\phi(x)-\phi(y)|}{|x-y|} \leq K .
$$

Definition 1.3. Let $C$ and $C^{\prime}$ be clone Cantor sets in $\mathbb{R}^{n}$. If there exists a bi-Lipschitz map $\phi: \mathbb{R}^{n} \rightarrow \mathbb{R}^{n}$ such that $\phi(C)=C^{\prime}$, then $C$ is said to be bi-Lipschitz equivalent to $C^{\prime}$. We denote this by $C \stackrel{\phi}{\simeq} C^{\prime}$.

The Cantor set $C$ has the topology induced by that of $\mathbb{R}^{n}$. Thus we can speak of open and closed subsets of $C$. In particular, by a clopen, we mean a subset of $C$ which is both open and closed as a subset of $C$ with respect to the induced topology. It is obvious that every clone of $C$ is a clopen; one can also see that every clopen is a union of finitely many clones.

In [9] we showed that if $\mathcal{I}$ is an $n$-dimensional cube and if $C(\tau)=\left\{\tau_{i}\right\}_{i=1}^{q}$ and $C\left(\tau^{\prime}\right)=\left\{\tau_{i}^{\prime}\right\}_{i=1}^{q}$ are clone Cantor sets in $\mathbb{R}^{n}, n \geq 2$, such that for all $i$ the maps $\tau_{i}^{-1} \circ \tau_{i}^{\prime}$ are in $S O(n)$, then $C(\tau)$ is bi-Lipschitz equivalent to $C\left(\tau^{\prime}\right)$. This operation of changing $C$ to $C^{\prime}$ is called sliding. A second method for 
constructing bi-Lipschitz equivalent clone Cantor sets is to change the choice of level-1 clones in some given clone Cantor set $C$. This is called splitting. The new Cantor set $C^{\prime}$ is identical to $C$, but has different level- 1 clones. When combined with sliding the level-1 clones, this produces new bi-Lipschitz equivalences. The clone maps $\tau_{i}^{\prime}$ for $C^{\prime}$ are certain compositions of the clone maps, $\tau_{i}$, for $C$. The operations of splitting and sliding generate an equivalence relation on the set of clone Cantor sets in $\mathbb{R}^{n}$.

The following classification result is proved in [9].

Theorem 1.4. Suppose that $n \geq 2$. Consider clone Cantor sets for the case that $\mathcal{I}$ is the unit cube in $\mathbb{R}^{n}$. If $C(\tau)=\left\{\tau_{i}\right\}_{i=1}^{q}$ is a clone Cantor set in $\mathbb{R}^{n}$ such that the collection of numbers

$$
\left\{\mu_{C}\left(A_{i}\right) / \mu_{C}\left(A_{1}\right): \quad A_{i} \text { is a level-1 clones of } C \quad 2 \leq i \leq q\right\}
$$

is algebraically independent over $\mathbb{Q}$, then any other clone Cantor set bi-Lipschitz equivalent to $C$ is equivalent to $C$ under splitting and sliding.

We now give a brief analysis of Hausdorff measure and dimension on these clone Cantor sets.

\subsection{Hausdorff Measure and Dimension on Clone Cantor Sets}

Let $C(\tau)=\left\{\tau_{i}\right\}_{i=1}^{q}$ be a clone Cantor set in $\mathbb{R}^{n}$. As in (1), express each $\tau_{i}$ as

$$
\tau_{i}(x)=\lambda_{i} T_{i}(x)+\vec{x}_{i} \quad \text { for some } \lambda_{i} \in \mathbb{R}^{+}, T_{i} \in O(n) \text {, and } \vec{x}_{i} \in \mathbb{R}^{n} .
$$

Then the Hausdorff dimension of $C, \operatorname{dim}_{H}(C)$, is that $d \in(0, n)$ satisfying the equation

$$
\sum_{i=1}^{q} \frac{1}{\lambda_{i}^{d}}=1
$$

Alternatively, if the collection of level-1 clones $\left\{A_{i}\right\}_{i=1}^{q}$ is given instead of the level-1 clone maps $\left\{\tau_{i}\right\}_{i=1}^{q}$, the Hausdorff dimension of $C$ is that $d \in(0, n)$ satisfying the equation

$$
\sum_{i=1}^{q}\left|A_{i}\right|^{d}=(|\mathcal{I}|)^{d} \quad \text { where }\left|A_{i}\right|=\operatorname{diam}\left(A_{i}\right) .
$$

Furthermore, if $\mu_{C}$ is the associated Hausdorff $d$-dimensional measure on $C$, then $0<\mu_{C}(C)<\infty$. Hence, $\mu_{C}$ can be normalized to a probability measure on $C$. See [4, Theorem 9.3]. 
Assuming that $\mu_{C}$ is normalized to a probability measure on $C$, then

$$
\mu_{C}(A)=\left(\frac{|A|}{|\mathcal{I}|}\right)^{d} \quad \text { for every clone } A \text { of } C .
$$

Note that the Hausdorff dimension of $C$ and the Hausdorff measure of its clones are dependent only on the sizes of $\mathcal{I}$ and the level-1 clones of $C$ and not on the similarities that generate the set.

\section{Main Results}

In this section, we will show that a bi-Lipschitz map from one clone Cantor set to another induces a bi-Lipschitz map, possibly no longer surjective, but having constant Radon-Nikodym derivative with respect to the associated Hausdorff measures on the clone Cantor sets. With this result in hand, we proceed to develop an invariant for these clone Cantor sets under bi-Lipschitz maps called the clopen invariant. Briefly, a clone Cantor set $C$ contains countably many clopens. The clopen invariant is the collection of the measures of these clopens using the associated Hausdorff measure of $C$. Another invariant of biLipschitz equivalence is the collection of scale factors at local similarity points. A local similarity point $x$ of $C$ is the fixed point of a local similarity. These are generalizations of clone maps. We obtain information on the contracting (expanding) factor of the local similarities.

\subsection{Measure Linearity}

Definition 2.1. Let $\left(X, \mu_{X}\right),\left(Y, \mu_{Y}\right)$ be measure spaces. A map $\phi: X \rightarrow Y$ is measure linear if there exists a constant $\lambda>0$ such that for all $\mu_{X}$-measurable sets $B \subseteq X, \phi(B)$ is $\mu_{Y}$-measurable and $\mu_{Y}(\phi B)=\lambda \mu_{X}(B)$.

The next theorem is our main result.

Theorem 2.2. Let $C$ and $C^{\prime}$ be clone Cantor sets in $\mathbb{R}^{n}$. If $\phi: \mathbb{R}^{n} \rightarrow \mathbb{R}^{n}$ is a bi-Lipschitz map such that $\phi(C)$ is a clopen subset of $C^{\prime}$, then there exists a clone $A$ of $C$ on which $\left.\phi\right|_{A}$ is measure linear with respect to the measure spaces $\left(C, \mu_{C}\right)$ and $\left(C^{\prime}, \mu_{C^{\prime}}\right)$.

The theorem states that by restricting the bi-Lipschitz map $\phi$ to a sufficiently small subset $A$ of $C$, the behavior of $\phi$ is linear at the measure level on $A$; moreover, $A$ is a clone of $C$, i.e., $A$ is a linearly scaled down copy of $C$.

For the proof of Theorem 2.2, we need the following definition and several preliminary lemmas. 
Definition 2.3. Let $C$ be a clone Cantor set in $\mathbb{R}^{n}$. For any subset $A$ of $C$ and a similarity $\sigma: \mathbb{R}^{n} \rightarrow \mathbb{R}^{n}$, the separation of $(\sigma, A)$ is defined by

$$
\operatorname{sep}(\sigma, A) \equiv d(\sigma(A), \sigma(C \backslash A)),
$$

the distance between $\sigma(A)$ and $\sigma(C \backslash A)$. For convenience, we use the notation $\operatorname{sep}(A)$ to mean $\operatorname{sep}(\mathrm{id}, A)$.

Remark 2.4. Note that

$$
\frac{\operatorname{sep}(A)}{\operatorname{diam}(A)}=\frac{\operatorname{sep}(\sigma, A)}{\operatorname{diam}(\sigma(A))}
$$

In particular, if $\tau: B \rightarrow \mathcal{I}$ is a level- $k$ clone map of $C, \tau$ extends uniquely to a similarity $\tau^{*}: \mathbb{R}^{n} \rightarrow \mathbb{R}^{n}$ and it follows that

$$
\frac{\operatorname{sep}(A)}{\operatorname{diam}(A)}=\frac{\operatorname{sep}\left(\tau^{*}, A\right)}{\operatorname{diam}\left(\tau^{*}(A)\right)} .
$$

Because of the uniqueness of this extension, for ease of notation, when necessary, we will treat a level- $k$ clone map $\tau$ of $C$ as a similarity on $\mathbb{R}^{n}$, i.e., we will retain the notation $\tau$ instead of $\tau^{*}$.

Lemma 2.5. Let $C$ be a clone Cantor set in $\mathbb{R}^{n}$ and let $A$ be a subset of $C$. If $B$ is the smallest clone of $C$ containing $A$, then $\operatorname{diam}(A) \geq \beta$ where

$$
\beta=\min \left\{\operatorname{sep}\left(B_{i}\right): B_{i} \text { is a relative level-1 clone of } B\right\} .
$$

Proof. It is readily seen that if $\operatorname{diam}(A)<\beta$, then $A$ is contained in some relative level-1 clone $B_{j}$ of $B$, which contradicts the hypothesis that $B$ is the smallest clone of $C$ containing $A$.

Lemma 2.6. Suppose that $C$ and $C^{\prime}$ are clone Cantor sets. Suppose that $\phi: R^{n} \rightarrow R^{n}$ is bi-Lipschitz and that $\phi C$ is a clopen subset of $C^{\prime}$. For any clone $D$ of $C$, if $D^{\prime}$ is the smallest clone of $C^{\prime}$ that contains $\phi(D)$, and $\sigma: D^{\prime} \rightarrow \mathcal{I}$ is the corresponding clone map taking the clone $D^{\prime}$ onto $C^{\prime}$, then $\operatorname{sep}(\sigma \circ \phi, D)$ is bounded below independently of the choice of clone D.

Proof. First, we need to produce some preliminary constants. Let $A_{1}, A_{2}, \ldots$, $A_{q}$ be level-1 clones of $C$. Define

$$
\alpha \equiv \min _{1 \leq i \leq q}\left\{\frac{\operatorname{sep}\left(A_{i}\right)}{\operatorname{diam}\left(A_{i}\right)}\right\} ;
$$


and set

$$
\beta \equiv \min \left\{\operatorname{sep}\left(\sigma, D_{i}^{\prime}\right): D_{i}^{\prime} \text { is a relative level-1 clone of } D^{\prime}\right\},
$$

which is clearly

$$
=\min \left\{\operatorname{sep}\left(A_{i}^{\prime}\right): A_{i}^{\prime} \text { is a level-1 clone of } C^{\prime}\right\}
$$

because $\sigma$ "rescales" $D^{\prime}$ to $C^{\prime}$, and $\beta$ is the minimum of the separations of the corresponding relative level-1 clones.

Note that $\operatorname{sep}(A) / \operatorname{diam}(A) \geq \alpha$ independently of any clone $A$ in $C$ because of Remark 2.4. We now proceed with the proof of the lemma.

Since $D$ is a clone of $C$, it is compact in $C$. Moreover, the bi-Lipschitz map $\phi$ is easily seen to be a homeomorphism so that the image $\phi(D)$ is compact in $C^{\prime}$, and hence is closed and bounded.

Therefore, we can find $x_{0}, x_{0}^{*} \in D$ so that

$$
\operatorname{diam}(\phi(D))=\left|\phi\left(x_{0}\right)-\phi\left(x_{0}^{*}\right)\right|
$$

Using a similar argument and the compactness of $C \backslash D$, we can also find an $x_{1} \in D$ and a $y_{1} \in C \backslash D$ satisfying the equality:

$$
\operatorname{sep}(\phi, D)=\left|\phi\left(x_{1}\right)-\phi\left(y_{1}\right)\right|
$$

If $K$ is the bi-Lipschitz constant of $\phi$, together with (2) and (3), we have

$$
\begin{aligned}
\frac{\operatorname{sep}(\sigma \circ \phi, D)}{\operatorname{diam}(\sigma \circ \phi(D))} & =\frac{\operatorname{sep}(\phi, D)}{\operatorname{diam}(\phi(D))} \\
& =\frac{\left|\phi\left(x_{1}\right)-\phi\left(y_{1}\right)\right|}{\left|\phi\left(x_{0}\right)-\phi\left(x_{0}^{*}\right)\right|} \\
& \geq \frac{K^{-1}\left|x_{1}-y_{1}\right|}{K\left|x_{0}-x_{0}^{*}\right|} \\
& \geq \frac{\alpha}{K^{2}} ;
\end{aligned}
$$

the first equality follows immediately because $\sigma$ is a similarity.

Now, by Lemma 2.5, we have

$$
\operatorname{sep}(\sigma \circ \phi, D) \geq \frac{\alpha \operatorname{diam}(\sigma \circ \phi(D))}{K^{2}} \geq \frac{\alpha \beta}{K^{2}} ;
$$

which is the conclusion we desired. 
Lemma 2.7. Suppose that $C$ and $C^{\prime}$ are clone Cantor sets in $\mathbb{R}^{n}$. Given $K>1$ there is an integer $N_{K} \geq 1$ and subsets $E_{1}^{\prime}, E_{2}^{\prime}, \ldots, E_{N_{K}}^{\prime}$ of $C^{\prime}$ with the following property. Suppose that $\phi: \mathbb{R}^{n} \rightarrow \mathbb{R}^{n}$ is a $K$ bi-Lipschitz map such that $\phi(C)$ is a clopen subset of $C^{\prime}$. Let $D^{\prime}$ be the smallest clone of $C^{\prime}$ containing $\phi(D)$. Let $\sigma: D^{\prime} \rightarrow \mathcal{I}$ the corresponding clone map of $D^{\prime}$ onto $C^{\prime}$. Then

$$
\sigma \circ \phi(D) \in\left\{E_{1}^{\prime}, E_{2}^{\prime}, \ldots, E_{N_{K}}^{\prime}\right\}
$$

Remark 2.8. The above lemma simply states that up to rescaling, there are only finitely many possibilities for the image of any clone $D$ of $C$ under $\phi$. Note that $N_{K}$ depends only on the constant $K$ and not on the bi-Lipschitz $\operatorname{map} \phi$.

Proof. Let $D$ be a clone of $C$ and hence a clopen of $C$. Therefore, it follows that $\sigma \circ \phi(D)$ is a clopen and thus is expressible as

$$
\sigma \circ \phi(D)=\bigcup_{i=1}^{r} C_{i}^{\prime}
$$

where each $C_{i}^{\prime}$ is a clone of $C^{\prime}$. Choose $p$ to be the smallest level such that $\sigma \circ \phi(D)$ is expressible as a union of finitely many level- $p$ clones of $C^{\prime}$. Note that the larger the level, the smaller the diameter of the clones. Hence, if $B^{\prime}$ is a level- $k$ clone of $C^{\prime}$, then $B^{\prime}$ is expressible as a union of finitely many level- $p$ clones of $C^{\prime}, k \leq p$. Now, by our choice of $p$, there exists a level- $(p-1)$ clone $B^{\prime}$ of $C^{\prime}$ such that

$$
B^{\prime} \cap \sigma \circ \phi(D) \neq \emptyset \quad \text { and } \quad B^{\prime} \cap \sigma \circ \phi(C \backslash D) \neq \emptyset ;
$$

so, by Lemma 2.6,

$$
\operatorname{diam}\left(B^{\prime}\right) \geq \operatorname{sep}(\sigma \circ \phi, D) \geq \frac{\alpha \beta}{K^{2}}
$$

We have shown that $\operatorname{diam}\left(B^{\prime}\right)$ is bounded below; thus, the level of $B^{\prime}$ is bounded above, so that $p$ is bounded above. Suppose that $p$ is bounded above by $l$. We can now conclude that

$$
\sigma \circ \phi(D)=\bigcup_{\text {finite }}\left(\text { level-l clones of } C^{\prime}\right) .
$$

As there are only finitely many level- $l$ clones of $C^{\prime}$, there are only finitely many such unions possible, which give rise to finitely many $E_{i}^{\prime}$ 's of $C^{\prime}$ with $\sigma \circ \phi(D)=E_{i}^{\prime}$ and the lemma is proved. 
Proof of Theorem 2.2. Since $\phi: \mathbb{R}^{n} \rightarrow \mathbb{R}^{n}$ is a bi-Lipschitz map with $\phi(C)$ a clopen subset of $C^{\prime}$, if $\operatorname{dim}_{H}(C)=d$, then $\operatorname{dim}_{H}\left(C^{\prime}\right)=d$. Moreover, for all $\mu_{C}$-measurable sets $B \subseteq C, \phi(B)$ is $\mu_{C^{\prime}}$-measurable. See [4, Theorem 9.3]. In particular, if $K$ is the bi-Lipschitz constant of $\phi$, we have

$$
K^{-d} \leq \frac{\mu_{C^{\prime}}(\phi(B))}{\mu_{C}(B)} \leq K^{d} .
$$

Now, for any subset $B \subseteq C$, define the mass ratio of $B$ to be

$$
\operatorname{MR}(B) \equiv \frac{\mu_{C^{\prime}}(\phi(B))}{\mu_{C}(B)},
$$

and set

$$
M \equiv \sup \{\operatorname{MR}(B): B \text { a clone of } C\} .
$$

Next, fix an $\epsilon>0$ and choose a clone $A$ of $C$ satisfying

$$
\operatorname{MR}(A)+\epsilon>M
$$

We will show that, for $\epsilon$ sufficiently small, $\left.\phi\right|_{A}$ is measure linear.

First, for every pair of measurable subsets $D$ and $E$ of $C$, define

$$
\rho(E, D) \equiv \frac{\operatorname{MR}(E)}{\operatorname{MR}(D)} .
$$

Claim 2.9. There exists a finite set $S \subset \mathbb{R}$, such that if $D$ is any clone and $E$ is relative level-1 clone in $D$, then $\rho(E, D) \in S$.

Proof of Claim. Let $D^{\prime}$ be the smallest clone of $C^{\prime}$ containing $\phi(D)$ and $\sigma: D^{\prime} \rightarrow C^{\prime}$ be the corresponding clone map. Examining $\rho(E, D)$ closely, we have the following equality.

$$
\begin{aligned}
\rho(E, D) & =\frac{\operatorname{MR}(E)}{\operatorname{MR}(D)} \\
& =\frac{\mu_{C^{\prime}}(\phi(E))}{\mu_{C}(E)} / \frac{\mu_{C^{\prime}}(\phi(D))}{\mu_{C}(D)} \\
& =\frac{\mu_{C^{\prime}}(\sigma \circ \phi(E))}{\mu_{C}(E)} / \frac{\mu_{C^{\prime}}(\sigma \circ \phi(D))}{\mu_{C}(D)} \\
& =\frac{\mu_{C^{\prime}}(\sigma \circ \phi(E))}{\mu_{C^{\prime}}(\sigma \circ \phi(D))} / \frac{\mu_{C}(D)}{\mu_{C}(E)} \\
& \equiv \delta / \gamma .
\end{aligned}
$$


Note that $\sigma \circ \phi(E)$ is well-defined since $\phi(E) \subseteq \phi(D) \subseteq D^{\prime}=$ domain $(\sigma)$; the third equality holds because $\sigma$ is a clone map.

Lemma 2.7 assures us that $\delta$ takes on only finitely many values. As for $\gamma$, if $A_{1}, A_{2}, \ldots, A_{q}$ are the level-1 clones of $C$, then, by the self-similarity property of $C$,

$$
\gamma \in\left\{\frac{\mu_{C}(C)}{\mu_{C}\left(A_{1}\right)}, \frac{\mu_{C}(C)}{\mu_{C}\left(A_{2}\right)}, \ldots, \frac{\mu_{C}(C)}{\mu_{C}\left(A_{q}\right)}\right\}
$$

which is clearly finite. Hence our claim follows.

Returning to the proof of the theorem, if $S=\{1\}$, then every clone has identical mass ratio, and thus, $\phi$ is measure linear on all of $C$. Otherwise, $S$ contains a number larger than 1 . The reason for this is readily seen from the following equation.

$$
1=\sum_{\substack{\text { level- } p \text { clones } \\ B \text { of } C}} \frac{\mu_{C^{\prime}}(\phi(B))}{\mu_{C^{\prime}}(\phi(C))}=\sum \rho(B, C) \frac{\mu_{C}(B)}{\mu_{C}(C)} .
$$

Observe that if $\rho(B, C) \leq 1$ for all level- $p$ clones $B$ of $C$, then $\rho(B, C)=1$ for all clones. Thus, taking our claim into consideration, we can define

$$
R \equiv \min \{r \in S: r>1\} .
$$

Intuitively, $R$ is the minimum amount of non-linearity which can occur at the measure level of $\phi$.

Now, if $\left.\phi\right|_{A}$ is not measure linear, then there is a clone $B \subset A$ with $\rho(B, A) \neq 1$. Let $p$ be the smallest level where this situation arises, i.e., there exists a level- $p$ clone $B \subset A$ with $\rho(B, A) \neq 1$, and no level- $(p-1)$ clone $B^{*} \subset A$ with $\rho\left(B^{*}, A\right) \neq 1$; then, using an identical argument to that of (4), there must be a level- $p$ clone $B^{\prime} \subset A$ with $\rho\left(B^{\prime}, A\right)>1$. Let $B^{\prime \prime}$ be the unique level- $(p-1)$ clone containing $B^{\prime}$, then $B^{\prime \prime} \subseteq A$ and $\rho\left(B^{\prime \prime}, A\right)=1$ by choice of $B^{\prime}$.

Next, consider the equality

$$
\rho\left(B^{\prime}, A\right)=\rho\left(B^{\prime}, B^{\prime \prime}\right) \rho\left(B^{\prime \prime}, A\right) .
$$

The equation $\rho\left(B^{\prime \prime}, A\right)=1$ implies that $\rho\left(B^{\prime}, A\right)=\rho\left(B^{\prime}, B^{\prime \prime}\right)>1$. Hence, $\rho\left(B^{\prime}, B^{\prime \prime}\right) \geq R$ and it follows that $\rho\left(B^{\prime}, A\right) \geq R$ which implies that $\operatorname{MR}\left(B^{\prime}\right) \geq$ $R \cdot \operatorname{MR}(A)$. If $\epsilon$ is chosen to be sufficiently small so that $R(M-\epsilon)>M$, then

$$
\operatorname{MR}\left(B^{\prime}\right) \geq R \cdot \operatorname{MR}(A)=R(M-\epsilon)>M,
$$

which contradicts our definition of $M$; therefore, $\left.\phi\right|_{A}$ must be measure linear. 
Remark 2.10. Let $C \stackrel{\phi}{\simeq} C^{\prime}$ be bi-Lipschitz equivalent clone Cantor sets in $\mathbb{R}^{n}$. Then, by Theorem 2.2, there exists a clone $A$ of $C$ such that $\left.\phi\right|_{A}: A \rightarrow C^{\prime}$ is measure linear. Let $\sigma: \mathbb{R}^{n} \rightarrow \mathbb{R}^{n}$ be the corresponding clone map taking $A$ onto $C$ (see Remark 2.4). Then the composition $\left.\phi\right|_{A} \circ \sigma^{-1}: \mathbb{R}^{n} \rightarrow \mathbb{R}^{n}$ gives us the following corollary.

Corollary 2.11. If $C \stackrel{\phi}{\simeq} C^{\prime}$ are bi-Lipschitz equivalent clone Cantor sets in $\mathbb{R}^{n}$, then $\phi$ induces a bi-Lipschitz map $\Phi: \mathbb{R}^{n} \rightarrow \mathbb{R}^{n}$ such that $\Phi(C)$ is a clopen subset of $C^{\prime}$ and $\Phi$ is measure linear everywhere on $C$ with respect to the Hausdorff measures on $C$ and $C^{\prime}$.

We also have the following Theorem, whose proof is technical and is omitted.

Theorem 2.12. Let $B$ be a clopen of a clone Cantor set $C=\left\{\tau_{i}\right\}_{i=1}^{q}$ in $\mathbb{R}^{n}, n \geq 2$. Then $B$ is bi-Lipschitz equivalent to $C$; i.e., there exists a biLipschitz map $F: \mathbb{R}^{n} \rightarrow \mathbb{R}^{n}$ such that $F(B)=C$.

Remark 2.13. For the case when $C$ is a subset of $\mathbb{R}$, see [2, Proposition 2.2]

We are now ready to develop an invariant for this class of clone Cantor sets under bi-Lipschitz equivalence.

\subsection{The Clopen Invariant}

Definition 2.14. Let $C$ be a clone Cantor set in $\mathbb{R}^{n}$. Consider $\left(C, \mu_{C}\right)$ as a probability space. Then the clopen invariant of $C$ is defined to be the countable subset of $[0,1]$ given by

$$
\mathrm{CL}(C) \equiv\left\{\mu_{C}(A): A \text { is a clopen of } C\right\} .
$$

The following corollary is an immediate consequence of Corollary 2.11.

Corollary 2.15. If $C \stackrel{\phi}{\simeq} C^{\prime}$ are bi-Lipschitz equivalent clone Cantor sets in $\mathbb{R}^{n}$, then there exist constants $\alpha, \beta>0$ such that

$$
\alpha C L(C) \equiv\{\alpha x: x \in C L(C)\} \subseteq C L\left(C^{\prime}\right),
$$

and

$$
\beta C L\left(C^{\prime}\right) \subseteq C L(C)
$$




\subsection{Local Similarity}

In this section, we define the concept of local similarities and local similarity points. Using a standard technique called symbolic dynamics, we then show that each clone Cantor set contains a countably dense set of local similarity points. If we replace the original bi-Lipschitz map by the induced measure linear bi-Lipschitz map as provided by Corollary 2.11, then the collection of similarity points is invariant under the induced bi-Lipschitz map. We also deduce a necessary condition on the contracting (expanding) factor of the associated similarities.

Definition 2.16. The symbol space on $q$ symbols is

$$
\Sigma_{q} \equiv\{1,2, \ldots, q\}^{\mathbb{N}}=\left\{s=\left(s_{0} s_{1} s_{2} \ldots\right): 1 \leq s_{i} \leq q\right\} .
$$

Define a metric, $d_{\Sigma}$, on $\Sigma_{q}$ as follows. Given $s=\left(s_{0} s_{1} s_{2} \ldots\right), t=\left(t_{0} t_{1} t_{2} \ldots\right)$ in $\Sigma_{q}$ set

$$
d_{\Sigma}(s, t)=\sum_{i=0}^{\infty} \frac{\left|s_{i}-t_{i}\right|}{2^{i}} .
$$

Then $\left(\Sigma_{q}, d_{\Sigma}\right)$ becomes a totally disconnected compact metric space. The proof of this fact and all of the following assertions concerning symbolic dynamics can be found in [5] or [10].

Define the shift-operator $\sigma: \Sigma_{q} \rightarrow \Sigma_{q}$ by

$$
\sigma\left(s_{0} s_{1} s_{2} \ldots\right)=\left(s_{1} s_{2} s_{3} \ldots\right) .
$$

Then $\sigma$ is continuous.

Definition 2.17. A point $x$ in $\Sigma_{q}$ is an eventually periodic point of period $p$ of $\sigma$ if $p$ is the smallest positive integer for which there exists an integer $m \geq 0$ such that $\sigma^{p+i}(x)=\sigma^{i}(x)$ for all $i \geq m$.

We have the following proposition.

Proposition 2.18. 1. $\operatorname{Per}(\sigma) \equiv\left\{x \in \Sigma_{q}: x\right.$ is a periodic point of $\left.\sigma\right\}$ is countably dense in $\Sigma_{q}$.

2. There exists a dense orbit for $\sigma$ in $\Sigma_{q}$; i.e., there exists an $x$ in $\Sigma_{q}$ such that $\left\{\sigma^{n}(x): n \in \mathbb{Z}\right\}$ is dense in $\Sigma_{q}$.

We now establish the relationship between the dynamics of $\sigma$ on $\Sigma_{q}$ and $\tau$ on $C$. 
Definition 2.19. The itinerary of $x \in C$ is a sequence $S(x)=\left(s_{0} s_{1} s_{2} \ldots\right)$ where $s_{i}=j$ if $\tau^{i}(x) \in A_{j}$. (Recall that $A_{j}=\tau_{j}^{-1}(\mathcal{I})$.)

Theorem 2.20. Let $C=\left\{\tau_{i}\right\}_{i=1}^{q}$ be a clone Cantor set in $\mathbb{R}^{n}$. The map $S: C \rightarrow \Sigma_{q}$ is a homeomorphism such that the following diagram commutes:

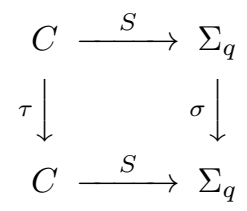

i.e., $S$ is a topological conjugacy between $\sigma$ and $\left.\tau\right|_{C}$.

Remark 2.21. Maps that are topologically conjugate are completely equivalent in terms of their dynamics. See [3]. With regard to our clone Cantor set $C$, the homeomorphism $S$ provides a one-to-one correspondence between the periodic points, eventually periodic points, orbits of $\left.\tau\right|_{C}$ and the corresponding points for $\sigma$.

Definition 2.22. A local similarity of a clone Cantor set $C$ in $\mathbb{R}^{n}$ is a homeomorphism $\sigma: U \rightarrow U$ defined on some open set $U$ in $\mathbb{R}^{n}$ with $U \cap C \neq \emptyset$ and such that

1. $\sigma(U \cap C) \subset C$.

2. $\sigma$ has a unique fixed point $x$, and $x \in C$. We call $x$ a local similarity point.

3. $\sigma$ is measure linear.

4. Either $\sigma$ or $\sigma^{-1}$ (if $\sigma$ is expanding) has bounded distortion, or is $B D$; i.e., there exists a constant $K>1$ and Euclidean similarities $f_{i}$ and $K$-biLipschitz homeomorphisms $\psi_{i}$ such that each of the maps $\sigma^{i} \circ \psi_{i} \circ f_{i} \circ \psi_{i}^{-1}$ is a $K$-bi-Lipschitz map.

Proposition 2.23. Let $C=\left\{\tau_{i}\right\}_{i=1}^{q}$ be a clone Cantor set in $\mathbb{R}^{n}$. All eventually periodic points of $\tau=\bigcup_{i=1}^{q} \tau_{i}$ are local similarity points of $C$.

Proof. Let $x \in C$ be an eventually periodic point of period $p$ of $\tau$; then there exists a constant $m \geq 0$ such that $\tau^{p+m}(x)=\tau^{m}(x)$. In particular, $\tau^{m} x$ is a similarity point of period $p$. The map $\tau^{m}$ maps a neighborhood of $x$ to a neighborhood of $\tau^{m} x$ and thus conjugates the local similarity at the point $\tau^{m} x$ to one at $x$.

Combining Proposition 2.23 with Theorem 2.20, we have the following result. 
Proposition 2.24. $S_{C} \equiv\{x \in C: x$ is a local similarity point of $C\}$ is a countably dense subset of $C$.

Remark 2.25. Note that the level-1 clone maps $\tau_{i}$ 's are local similarities of $C$ with $\operatorname{domain}\left(\tau_{i}\right)=A_{i}$.

Proposition 2.26. Suppose that $C$ and $C^{\prime}$ are clone Cantor sets. Suppose that $\phi$ bi-Lipschitz homeomorphism of $\mathbb{R}^{n}$ mapping $C$ onto a clopen in $C^{\prime}$. Suppose that $\phi$ is is a measure-linear with respect to Hausdorff measure on $C$ and $C^{\prime}$. If $x$ is a local similarity point of $C$, then $\phi(x)$ is a local similarity point of $C^{\prime}$.

Proof. Suppose that $x$ is a local similarity point of $C$ with associated local similarity $\sigma$; then $\sigma^{\prime} \equiv \phi \circ \sigma \circ \phi^{-1}$ is $\mathrm{BD}$ because $\sigma$ is $\mathrm{BD}$ and $\phi$ is biLipschitz. It's also immediate that $\sigma^{\prime}$ is measure linear because both $\sigma$ and $\phi$ are measure linear. In fact, $d \sigma^{\prime} / d \mu_{C^{\prime}}=d \sigma / d \mu_{C}$, where $d \sigma^{\prime} / d \mu_{C^{\prime}}$ and $d \sigma / d \mu_{C}$ are the measure linear constants of $\sigma^{\prime}$ and $\sigma$ respectively. Hence, $\sigma^{\prime}$ is a local similarity of $C^{\prime}$ with corresponding local similarity point $\phi(x)$.

The following proposition states a necessary condition on the contracting (expanding) factors of local similarities of a clone Cantor set.

Proposition 2.27. Let $C=\left\{\tau_{i}\right\}_{i=1}^{q}$ be a clone Cantor set in $\mathbb{R}^{n}$. If $\sigma$ is an expanding local similarity of $C$ with $d \sigma / d \mu_{C}=\lambda$, then there exist constants $s, p \in \mathbb{N}$ such that $\lambda^{s}=\lambda_{i_{1}} \lambda_{i_{2}} \ldots \lambda_{i_{p}}, 1 \leq i_{j} \leq q$, where $\lambda_{i}=d \tau_{i} / d \mu_{C}$. If $\sigma$ is contracting, then $\lambda^{s}=1 / \lambda_{i_{1}} \lambda_{i_{2}} \ldots \lambda_{i_{p}}$.

Proof. We will prove the case when $\sigma$ is contracting. For the case when $\sigma$ is expanding, replace $\sigma$ by $\sigma^{-1}$.

Let $\sigma$ be a local similarity of $C$ with associated local similarity point $x$. Choose a clone $A \subset \operatorname{domain}(\sigma)$ such that $x \in A$. Since $\sigma$ is contracting, $\sigma^{P}(A) \subset A$ for sufficiently large $p>0$. We replace $\sigma$ with $\sigma^{p}$. Let $B_{r}=\sigma^{r}(A)$ and define $A_{r}$ to be the smallest clone of $C$ containing $B_{r}$. Because $\sigma$ is $\mathrm{BD}$, $\operatorname{sep}\left(\sigma^{r}, A\right) / \operatorname{diam}\left(A_{r}\right)$ is bounded below independent of $r$; then, as in the proof of Lemma 2.7, there exist finitely many possibilities for the image of $A$ under $\psi_{r} \circ \sigma^{r}$ where $\psi_{r}: A_{r} \rightarrow \mathcal{I}$ is the corresponding clone map taking $A_{r}$ onto $C$. Therefore, there exist $r, s \in \mathbb{N}$ such that $\psi_{r} \circ \sigma^{r}(A)=\psi_{r+s} \circ \sigma^{r+s}(A)$, or $\psi\left(B_{r}\right)=\psi_{r+s}\left(B_{r+s}\right)$.

Define $\gamma \equiv \psi_{r+s}^{-1} \circ \psi_{r}$. Then $\gamma\left(B_{r}\right)=B_{r+s}$. Now, since $\gamma$ and $\sigma^{s}$ are measure linear and $\sigma^{s}\left(B_{r}\right)=B_{r+s}=\gamma\left(B_{r}\right)$, we have

$$
\mu_{C}\left(\sigma^{s}\left(B_{r}\right)\right)=\mu_{C}\left(\gamma\left(B_{r}\right)\right) \quad \text { so that } \quad \lambda^{s}=\frac{d \gamma}{d \mu_{C}} .
$$


Let $m=$ level of $A_{r}$, and $p=\left(\right.$ level of $\left.A_{r+s}\right)-m$, then $\psi=\left.\tau^{m}\right|_{A_{r}}$ and $\psi_{r+s}=\left.\tau^{m+p}\right|_{A_{r+s}}$. Then,

$$
\frac{d \psi_{r}}{d \mu_{C}}=\lambda_{i_{1}} \lambda_{i_{2}} \ldots \lambda_{i_{m}}
$$

and

$$
\frac{d \psi_{r+s}}{d \mu_{C}}=\lambda_{i_{1}} \lambda_{i_{2}} \ldots \lambda_{i_{m}} \lambda_{i_{m+1}} \lambda_{i_{m+2}} \ldots \lambda_{i_{m+p}}
$$

where $1 \leq i_{j} \leq q$ and $\lambda_{i}=d \tau_{i} / d \mu_{C}$. Finally, assembling (5), (6), and (7), we have

$$
\lambda^{s}=\frac{1}{\lambda_{i_{m+1}} \lambda_{i_{m+2}} \ldots \lambda_{i_{m+p}}}
$$

and the proposition is proved.

\section{References}

[1] B. Farb, L. Mosher, D. Cooper, A Rigidity Theorem for the Solvable Baumslag-Solitar Groups. Duke J. Math, too appear.

[2] D. Cooper and T. Pignataro, On the shape of Cantor sets, J. Differential Geometry 28 (1988), 203-221.

[3] R. Devaney. Chaotic Dynamical Systems, The Benjamin/Cummings Publishing Company, Inc., 1986.

[4] K. Falconer, Fractal Geometry, John Wiley and Sons, 1990.

[5] M. Hata, Topological aspects of self-similar sets and singular functions, Fractal Geometry and Analysis (J. Bélair and S. Dubuc, eds.), Kluwer Academic Publishers, 1991, 255-276.

[6] J. E. Hutchinson, Fractals and self-similarity, Indiana Univ. Math. J. 30 (1981), 713-747.

[7] R. D. Mauldin and M. Urbański, Dimensions and Measures in Infinite Iterated Function Systems, Proc. London Math. Soc. (3) 73 (1996), 105154.

[8] C. A. Rogers, Hausdorff Measures, Cambridge University Press, 1970.

[9] H. Vuong, Classification of self-similar Cantor sets up to bi-Lipschitz equivalence, Ph.D. Thesis, University of California, Santa Barbara, CA, 1994.

[10] P. Walters, An Introduction to Ergodic Theory, Springer, 1982. 